TITLE:

\title{
An eClinical trial system for cancer that integrates with clinical pathways and electronic medical records.
}

\section{$\operatorname{AUTHOR}(S)$ :}

Yamamoto, Keiichi; Yamanaka, Kenya; Hatano, Etsuro; Sumi, Eriko; Ishii, Takamichi; Taura, Kojiro; Iguchi, Kohta; ... Yokode, Masayuki; Uemoto, Shinji; Fukushima, Masanori

\section{CITATION:}

Yamamoto, Keiichi ... [et al]. An eClinical trial system for cancer that integrates with clinical pathways and electronic medical records.. Clinical trials 2012, 9(4): 408-417

\section{ISSUE DATE:}

2012-08

URL:

http://hdl.handle.net/2433/178667

\section{RIGHT:}

(c) 2012 by The Society for Clinical Trials; この論文は出版社版でありま せん。引用の際には出版社版をご確認ご利用ください。; This is not the published version. Please cite only the published version. 


\section{An eClinical Trial System for Cancer that Integrates with Clinical Pathways and}

\section{Electronic Medical Records}

Running head: A New Integrated eClinical Trial System

Total number of words: 3,761

Keiichi Yamamoto $^{1 *}$, Kenya Yamanaka ${ }^{2 *}$, Etsuro Hatano ${ }^{2}$, Eriko Sumi ${ }^{3}$, Takamichi

Ishii $^{2}$, Kojiro Taura ${ }^{2}$, Kohta Iguchi $^{2}$, Satoshi Teramukai ${ }^{1}$, Masayuki Yokode ${ }^{3}$, Shinji

Uemoto $^{2}$, Masanori Fukushima ${ }^{4}$

${ }^{1}$ Department of Clinical Trial Design and Management, Translational Research Centre,

Kyoto University Hospital, Kyoto, Japan

${ }^{2}$ Department of Surgery, Graduate School of Medicine, Kyoto University, Kyoto, Japan

${ }^{3}$ Department of Clinical Innovative Medicine, Translational Research Centre, Kyoto

University Hospital, Kyoto, Japan

${ }^{4}$ Translational Research Informatics Centre, Foundation for Biomedical Research and

Innovation, Kobe, Japan

*These authors contributed equally to this paper. 
Corresponding author: Keiichi Yamamoto, 54 Shogoin Kawahara-cho, Sakyo-ku,

Kyoto, 606-8507 Japan. E-mail kyamamo@kuhp.kyoto-u.ac.jp

Tel +81-75-751-4717, Fax +81-75-751-3399 


\section{Abstract}

Background Various information technologies currently are used to improve the efficiency of clinical trials. However, electronic medical records (EMRs) are not yet linked to the electronic data capture (EDC) system. Therefore, the data must be extracted from medical records and transcribed to the EDC system. Clinical pathways are planned process patterns that are used in routine clinical practice and are easily applicable to the medical care and evaluation defined in a trial protocol. However, few clinical pathways are intended to increase the efficiency of clinical trials.

Purpose Our purpose is to describe the design and development of a new clinical trial process model that enables the primary use of EMRs in clinical trials by integrating clinical pathways and EMRs.

Methods We designed a new clinical trial model that uses EMR data directly in clinical trials and developed a system to follow this model. We applied the system to an investigator-initiated clinical trial and examined whether all data were extracted correctly. At the protocol development stage, our model measures endpoints based on 
clinical pathways with the same diagnosis. Next, medical record descriptions and the format of the statistical data are defined. According to these observations, screens to entry data which is used for both in clinical practice and for study are prepared into EMRs with an EMR template, and screens are prepared for data checks on our EMR retrieval system (ERS). In an actual trial, patients are registered and randomly assigned to a protocol treatment. The protocol treatment is executed according to clinical pathways, and the data are recorded to EMRs using EMR templates. The data are checked by a local data manager using reports created by the ERS. After edit checks and corrections, the data are extracted by the ERS, archived in portable document format (PDF) with an electronic signature, and transferred in comma separated values format (CSV) to a coordinating centre. At the coordinating centre, the data are checked, integrated, and made available for statistical analysis.

Results We verified that the data could be extracted correctly and found no unexpected problems.

Limitation To execute clinical trials in our system, the EMR template and efficient EMR retrieval systems are required. Additionally, to execute multi-institutional clinical 
trials, it is necessary to create templates appropriate for EMRs at all participating sites and for the coordinating centre to validate local templates and procedures.

Conclusion We proposed and pilot tested a new eClinical trial model. Because our model is integrated with routine documentation of clinical practice and clinical trials, redundant data entries were avoided and the burden on the investigator was minimised. The reengineering of the clinical trial process would facilitate the establishment of evidence in the future.

\section{Keywords}

clinical research informatics, electronic data capture, CDISC, EMR retrieval, template, data warehouse 


\section{BACKGROUND}

To improve the efficiency of clinical trials, evaluating and restructuring the information-capture process and the flow of data are critical [1-3]. In many clinical trials, paper-based case report forms (CRFs) are developed for each clinical trial protocol and information is transcribed from source documents, including medical records, to CRFs and from CRFs to the clinical data management system (CDMS). Thus, mistranscriptions and inconsistencies are inevitable in this dual transcription process [2, 4]. Various information technologies currently are used to improve the efficiency of clinical trials, including computerised registration systems, CDMS, and electronic data capture (EDC) systems, among others [2, 4-5]. Elsewhere, medical information technology recently has been promoted. The electronic medical record (EMR) system has been launched in approximately $20 \%$ of all institutions in Japan. However, EMRs are not yet linked to the EDC system for clinical research; therefore, the clinical trial staff must manually select the necessary information from EMRs and transcribe it into the EDC system [2]. Thus, the workload at each participating site has not changed with the transition from paper-based CRFs to EDC systems, and there has been no breakthrough in efficiency [2]. 
The president of the Clinical Data Interchange Standards Consortium (CDISC),

Rebecca Daniels Kush, named the next generation of clinical trials 'eClinical trials' [3].

She emphasized the importance of optimising clinical trials by redesigning the trial process to utilise primarily electronic processes and to coordinate hospital information systems with the various clinical trials systems [3]. In addition, the CDISC's Electronic Source Data Interchange (eSDI) initiative, encouraged by the US Food and Drug Administration, was started in 2004 [4]. The eSDI detailed the regulatory requirements and outlined promising scenarios concerning clinical trials using electronic source data (eSource), such as electronic subject diaries, electronic laboratory results, and EMRs. To enhance clinical trials utilising eSource, the eSDI analysed the present regulatory requirements, compiled 12 indispensable requirements, outlined five scenarios that would fit the regulatory requirements, and formed a proposal.

Currently, most clinical trials including standards-based approaches that use data from EMRs are planned according to the concept that the primary use of EMRs is in clinical practice and a secondary use is clinical research [6-11]. To use EMR data secondarily in a clinical trial, it is necessary to obtain various types of information that are stored in EMRs by category, such as the diagnosis and medications taken. However, extraction of 
this information is not facilitated by the current EMR system [11-13]. Moreover, not all information that is necessary to execute a clinical trial typically is stored in the EMR [6, 10-12]. If EMRs are used only for the purpose of collecting certain receipts of claims and the treatments for each patient, enough information may be stored in the current EMRs. However, if there is a secondary intention to use the accumulated information from EMRs in clinical research, then the data that are not gathered specifically for research purposes may be incomplete and unreliable [6, 11-12]. To improve the efficiency of clinical trials, it is necessary to establish a method that utilises EMRs directly in clinical trials and enables higher levels of information-sharing and exchange between clinical practice and clinical trials [1-3].

Clinical pathways have been introduced in clinical practice $[14,15]$. Clinical pathways are planned process patterns aimed at improving both process quality and resource usage $[14,15]$. Clinical pathways include components such as a timeline, the categories of care or activities, and the interventions for specified groups of patients with a particular diagnosis. They are widely used as a treatment plan or as a substitute for physicians' orders $[14,15]$. Clinical pathways appear to be easily applicable to the protocol treatment of a clinical trial because they deliver a standard of care to patients with a specific disease 
$[14,15]$. However, there are few clinical pathways that purport to improve the efficiency of clinical trials.

\section{PURPOSE}

Our purpose is to develop a new clinical trial process model that enables the primary use of EMRs in a clinical trial by integrating clinical pathways and EMRs. We report herein on a pilot test of our model in a single-centre clinical trial conducted at our institution.

\section{METHODS}

Methods and outline of our model of the clinical trial process

We modelled a new clinical trial process in which practical medical records are used directly in clinical trials and developed a clinical trial system to follow our model. We applied the new system to an investigator-initiated clinical trial and examined whether all of the data needed to conduct the clinical trial were extracted correctly throughout the system. Moreover, by comparing the new model with the conventional method of 
conducting clinical trials, we made a qualitative observation of how the new model reduces the workload in clinical trials.

In our model, a clinical trial is performed as follows (see also Figure 1):

1. In the protocol development stage, the methods of measuring endpoints (i.e., protocol treatment and evaluation items) are decided based on the clinical pathways that usually are used for patients with the same diagnosis. Next, medical record descriptions are defined, including eligibility criteria, tests, protocol treatment, efficacy of treatment, adverse events and follow-up. At the same time, the data format and coding conventions for the statistical data are defined to be compatible with statistical software. For example, the description of adverse events is synchronised and standardised with clinical practice and clinical trials according to the Common Terminology Criteria for Adverse Events (CTCAE) [16].

2. According to the defined descriptions, screens are prepared for entry of the additional data required for the clinical trial into EMRs using EMR templates, and screens are prepared for data checks and data extraction by our EMR retrieval system (ERS). The templates are named to enable clinicians to identify them as pertaining only to the trial. 
3. Patients who provide informed consent and agree to participate are registered and randomly assigned to one of the protocol treatments.

4. The protocol treatment assigned to the patient is executed according to clinical pathways, and the treatment data are recorded to EMRs using EMR templates.

5. Data are extracted using the ERS and are checked automatically for completeness and consistency and to identify anomalies to be resolved.

6. After edit checks have been satisfied following corrections, the data are extracted by the ERS, archived in portable document format (PDF) with an electronic signature, and transferred in comma separated values format (CSV) to a coordinating centre.

7. At the coordinating centre, the clinical trial data from participating sites are checked, integrated, and made available for statistical analysis.

When it is necessary to update trial data that already have been transferred to the coordinating centre, the process repeats from when the investigator updates the medical records using the clinical trials template. In addition, central pathology reviews or central laboratory findings are integrated with clinical data in the coordinating centre. 
Because our new model is integrated, with the routine documentation of clinical practice and the procedures of clinical trials, to avoid redundant data entries, the efficiency of clinical trials can be improved.

\section{Applied information technology}

\section{EMR templates}

To record clinical trial data to EMRs that could be available for clinical practice, we used EMR template technology [14, 17-21].

In the current EMR system in Japan, various types of information are integrated, including coded data. Coded data include laboratory results that are in the computerised physician order entry system, narrative information, such as observations during physical examinations or progress notes that are written in free-text form, radiological images, and waveform information from electrocardiograms. In particular, it was necessary to convert free-text information to structured information that is expressed by data elements consisting of items and values with codes for use in a clinical trial [17]. The most practical method is to use a template for entering data into the EMR system 
[17]. Using templates, the entered narrative information can be made available for data analysis.

In Japan, the EMR template technology already has been implemented for most large hospitals' EMR systems, including ours, and is widely used to standardise medical records. Although multiple vendors supply various templates, when the new information is recorded, our hospital stores key data, such as the template ID, item ID, and entry date and time, in the EMR database. Moreover, our EMR system is managed according to the Japanese Ministry of Health, Labour and Welfare's guidelines on hospital information system management. To guarantee data integrity, all modifications to EMR data are recorded when and by whom each operation is executed, and an audit trail is made automatically. Audit trails record whose, when, and by whom medical records have been accessed. No user can update audit trails or prevent recording them. In addition, after medical records are submitted by medical staff, no user can delete the records. When medical records are updated, the new data are created as updated records, and the old data are retained as deleted records with a 'deletion flag'. This traceability management function is applied to the clinical trial template; all clinical trial data and histories are stored on the EMR system. 


\section{EMR retrieval system}

We use the ERS that was developed in our translational research centre to extract data from EMRs, verify the completeness and consistency of trial data, and create output data for statistical analysis. The ERS can retrieve data from patients participating in the clinical trial comprehensively and efficiently. To retrieve and report the records in clinical practice directly as data for clinical research, we identified entities from EMRs that are useful for clinical research (i.e., data on patient demographics, diagnosis, physical examination, progress notes recorded in the EMR template, operative notes, laboratory tests, radiological or pathological studies, medications and injections, and other treatments). Next, we designed a multidimensional data model for EMR retrieval for clinical research $[11,22-25]$. To ensure that the data retrieval process is practical and independent from the structure of the EMR system, a data warehouse was created by extracting, transforming, and loading information from the EMR system. To retrieve and report information from multiple patients efficiently, an online analytical processing (OLAP) tool was installed [26]. The OLAP tool runs from an Internet browser and has the ability, in hypertext markup language (HTML), to report on information retrieved 
from the browser. The reports are created in various formats, such as PDF, CSV, and extensible markup language (XML). The ERS also was applied to the data recorded using the new template. We created reports for checking trial data and extracting data for statistical analysis.

\section{Application to a clinical trial}

We applied the system to a single-institution phase II trial for hepatocellular carcinoma.

('A randomised controlled study of effectiveness between transcatheter arterial chemoembolisation (TACE) with cisplatin and TACE with epirubicin for multiple hepatocellular carcinomas'). This study was approved by the ethics committee of Kyoto University Hospital and was registered in the UMIN Clinical Trials Registry [27] (registration number UMIN000003162).

Based on the findings from an observational study in our hospital [28, 29], this phase II trial was designed to confirm the effectiveness of TACE in combination with one of the two anticancer agents. A total of 160 subjects will be enrolled, treated by a single TACE therapy, and evaluated for 12 months. Including laboratory tests, the number of data 
items per subject is approximately 80 , and the number of total records per subject is approximately 15 (Table 1).

\section{Procedures of the clinical trial}

\section{Preparation}

In the pilot test of our clinical trial process model, we prepared seven templates:

Eligibility Criteria, Target Lesion, Protocol Treatment, Efficacy of Treatment based on the European Association for the Study of the Liver (EASL) standard [30], Efficacy of Treatment based on the Response Evaluation Criteria in Cancer of the Liver (RECICL) standard [31], Adverse Events based on CTCAE 4.0, and Follow-Up (Table 1). Codes and grades (i.e., EASL, RECIEL, CTCAE) were assigned by the investigators when they entered these data into the EMR using the clinical trial templates. Three types of ERS reports were used to check the clinical trial data: a List of Cases, each patient's Case Registration Form, and the Case Report Form (Table 1). We show an example of the EMR template and progress notes from the EMR in Figure 2. 
For the local data manager to verify the completeness and consistency of trial data, the

Case Report Form can be identified. The Case Report Form summarises all the templates on one screen such that the integrity of all information for each patient can be assessed at a glance. The latest data for Eligibility Criteria, Target Lesion, Protocol Treatment, Efficacy of Treatment, and Follow-Up are displayed by item; Adverse Events are shown in sequence according to the name of the adverse event so that changes in the adverse event grade can be observed (Figure 3).

Patient registration and random assignment of treatment arm

Patients were registered and assigned randomly to a treatment arm in an independent registration centre outside of the EMR system. The investigator received the registration and assignment information and recorded it on the Eligibility Criteria template in the EMRs. 
The investigator conducted the protocol treatment and evaluations according to the

clinical pathways and recorded the results in templates. The results of required laboratory tests were extracted directly from the EMRs.

\section{Correction of data}

Clinical trials often require local-level administrative help with the increased workload $[5,32]$, but our model provides the local data manager with new responsibilities to ensure trial data quality. In the pilot study, a local data manager - a clinician at the participating site - manually checked the trial data for completeness and consistency using the Case Report Form on the ERS. When the local data manager found incomplete or inconsistent trial data, he asked the investigator to determine whether data should be corrected. The investigator added necessary data using the original EMR templates, thus storing a revision history in the EMRs.

In the pilot study, the data in the EMR system create the edit checks and format the data for statistical analysis; thus a separate EDC system or the CDMS is unnecessary. We executed data checks manually to ensure that the latest medical records were correct. 


\section{Extracting data for statistical analysis}

We used the ERS to extract data for the statistical analyses according to predefined data formatting and coding conventions. After the data were presented for statistical analysis in the CSV by the ERS, reports of all the data were prepared and archived in PDFs with an electronic signature added to prevent manipulation.

\section{RESULTS}

We applied our new system to an actual clinical trial and evaluated its feasibility.

Using the list of registered patients in the registration centre, we determined whether we could extract both the data by templates and the laboratory results of the registered patients in pre-designed forms. We verified that the data could be extracted correctly and found no unexpected problems. 
Regarding the clinical staff workload, the investigators did not need to complete CRFs or enter data into an EDC system in addition to the EMRs. Among the prepared templates for the clinical trial, the only one that was added to the pilot study was Eligibility Criteria for patient registration. The investigators had to record information in the medical records for TACE, including the number of tumours, their diameter and extent of vascular invasion, and the therapeutic regimen, such as anticancer drug doses and embolised arteries in the liver. To record the necessary clinical information on a patient's EMR, investigators used the templates based on the clinical pathways for TACE, and they checked the results of laboratory tests shown in the EMRs.

Regarding the data quality control, as the data in the EMR templates were identical to those in the ERS, the use of the EDC system or the CDMS was also unnecessary. When investigators added information to EMRs, local data managers viewed reports in the Case Report Form automatically on the ERS. Local data managers check the data until all of the errors are corrected. In this way, the progress of the clinical trial and the situation for each patient could be assessed at a glance. 
Figure 4 shows the procedural differences among a paper-based clinical trial, a clinical trial using the EDC system, and our new model. In contrast with conventional clinical trials, our model made it unnecessary to transcribe data from medical records to CRFs, to execute source data verification (SDV) at the participating site, to transport paper CRFs from the participating site to the coordinating centre, or to make double entries in the CDMS from paper CRFs. In addition, data checking was performed by the local data manager at the participating site, and supplemental data checking and data integration were performed at the central coordinating centre.

\section{LIMITATIONS}

To execute clinical trials according to our model, specialised data must be accumulated from EMRs according to each clinical trial protocol. From a technical viewpoint, equipment for the flexible input functions, such as the EMR template, and efficient EMR retrieval systems are required. Consequently, trials must be planned from the stage of protocol development. The pilot test trial was planned to answer the practical questions that clinicians face in clinical practice. If a clinical trial is not practice-based [33-35], then it will not be easy to add medical records using a template. In our model, 
the coordinating centre workload for data management and quality monitoring would be reduced. Instead, the workload of the local data manager at the participating site would be increased. In addition, in the test trial, every time errors were found in trial data using the Case Report Form on the ERS, the local data manager had to communicate with the investigator. In the future, more efficient local data-management methods will have to be established [36].

Because the test trial used for the pilot study was executed in a single institution, it was not necessary to integrate the clinical trial data from multiple participating sites. We used the CSV format to present data for statistical analysis and the PDF format to archive the trial data at the participating site. To execute multi-institutional clinical trials, it will be necessary to standardise the descriptions in EMRs, use evidence-based clinical pathways, and create and validate templates across EMRs at all participating sites [37]. Currently, the accuracy of the information in EMRs may vary across sites [12]. For example, there are standard medical terminologies, such as ICD9, ICD10 [38], MedDRA [39], and SNOMED [40], but diagnosis or medications are associated with the payment of medical insurance, and use of classification schemas and terminology are at the discretion of clinicians at each site. Thus, the central coordinating centre will 
need to monitor and standardise the use of EMRs and integrate data provided across sites. In addition, a vendor-neutral and platform-independent standard format, such as the CDISC Operational Data Model (ODM) [41] and the CDISC Study Data Tabulation Model (SDTM) [42], will be needed to archive, transfer, and integrate trial data. The advantage is that the EMR system would not need to be retained in the future to access the data or the audit trail [4].

\section{CONCLUSION}

We proposed a new clinical trial process model by integrating it with clinical pathways and EMRs, which is the primary use of EMRs in a clinical trial. In a single-centre trial selected for the pilot study, our new model was integrated with the routine documentation of clinical practice and the procedures of clinical trials. Therefore, redundant data entries for the trial were avoided, and the burden on the investigators posed by the trial was minimised.

The basis of our model is that the standardisation of clinical practice from clinical pathways can be applied as a treatment plan for a clinical trial; one primary use of the 
EMR system could be to provide data for clinical trials. The most prominent

characteristic of our model is that many data management tasks that typically are performed by the central coordinating centre are moved to the participating sites. In effect, the EMRs replace the EDC system and the CDMS. To maintain efficient data quality control, it is important to execute data management near the site of the data occurrence. The earlier an error is detected in a clinical trial, the sooner and more easily and cheaply it can be rectified [3]. Conventionally, the period from when an investigator executes protocol treatment to when the trial data are transcribed in the CRFs and checked by the data manager of the central coordinating centre spans several days (e.g., mean 5 days ranging 1 to 25 in our centre). In contrast, when investigators added information to the EMRs in the test trial, the data were immediately available to the local data manager for checking, which allowed rapid resolution of any anomalies introduced. Such real-time availability of trial data should reduce the time and cost of conducting clinical trials.

In addition, in the eSDI proposal, the third scenario was called 'Single Source Concept [4],: data need to be entered only once for multiple purposes (research, patient care, safety surveillance) within the context of existing regulations. The fourth scenario was 
called 'EMR Extraction and Investigator Verification': a clinician would check EMRs for the necessary data for the clinical trial and extract and transfer the data to a sponsor. We believe that our new model fits these scenarios and that we have demonstrated an implementation model for eClinical trials based on the eSDI proposal. According to the eSDI scenario, Title 21 Part 11 of the Code of Federal Regulations [43] starts at the point of the creation of a clinical research record. In other words, it is not necessary to apply these regulations to the medical records at the site. The clinical research record is created at the point of electronic signature signing. When data are extracted from EMRs as clinical trial data, the migration of the data from EMRs to the clinical trial database must be validated at the participating site. In addition, the sponsor has to produce documents on how the procedures of the clinical trial follow all appropriate regulations. Because of the direct extraction of the clinical trial data from EMRs, our model may eliminate or minimize the need for source data verification. The audit plan should focus on the accuracy of the migration of data from EMRs to ensure that data are not changed in the extraction process and that patient confidentiality requirements are met [4]. We believe that by appropriating the efforts of source data verification in favour of validating the clinical trial process, the costs can be reduced and the efficiency of clinical trials can be improved. 


\section{ACKNOWLEDGMENTS}

The authors would like to acknowledge the staff of the Department of Medical

Informatics of Kyoto University Hospital and the Kyoto University EBM Research

Centre for their generous support.

\section{REFERENCES}

1. Embi PJ, Payne PR. Clinical research informatics: challenges, opportunities and definition for an emerging domain. J Am Med Inform Assoc. 2009 May-Jun; $16(3): 316-27$

2. Zhengwu L and Jing S. Clinical data management: current status, challenges, and future directions from industry perspectives. Open Access J Clin Trials 2010; 2: 93-105.

3. Daniels Kush R. eClinical Trials: planning and implementation. Boston, MA: CenterWatch, 2003. p.12.p38

4. Clinical Data Interchange Standards Consortium (CDISC) 'Leveraging the CDISC standards to facilitate the use of Electronic Source Data within Clinical Trials 
(eSDI)

http://www.cdisc.org/stuff/contentmgr/files/0/2f6eca8f0df7caac5bbd4fadfd76d575

/miscdocs/esdi.pdf (2006, accessed June 2011).

5. McFadden E. Management of data in clinical trials. 2nd ed. Hoboken, NJ:

Wiley-Interscience, 2007. p33, p56, p80, p94.

6. Yamamoto K, Matsumoto S, Tada H, et al. A data capture system for outcomes studies that integrates with electronic health records: development and potential uses. J Med Syst 2008; 32(5): 423-427.

7. Kush R, Alschuler L, Ruggeri R, et all: Implementing Single Source: the STARBRITE proof-of-concept study. J Am Med Inform Assoc. 2007; 14(5):662-73

8. CDISC 'Healthcare Link Initiative', http://www.cdisc.org/healthcare-link (2009, accessed June 2011)

9. The ASTER Pilot Project 'Improving the reporting of adverse events', http://www.asterstudy.com/index.php?option=com_content\&view=article\&id=10: aster-description (2009, accessed June 2011). 
10. Yamamoto K, Matsumoto S, Yanagihara K, et al. A data-capture system for post-marketing surveillance of drugs that integrates with hospital electronic health records. Open Access J Clin Trials 2011; 3: 21-26.

11. Prokosch HU, Ganslandt T. Perspectives for medical informatics. Reusing the electronic medical record for clinical research. Methods Inf Med. 2009;48(1):38-44.

12. Wasserman RC. Electronic medical records (EMRs), epidemiology, and epistemology: reflections on EMRs and future pediatric clinical research. Acad Pediatr. 2011 Jul-Aug;11(4):280-7. Epub 2011 May 31.

13. Kristianson KJ, Ljunggren H, Gustafsson LL. Data extraction from a semi-structured electronic medical record system for outpatients: a model to facilitate the access and use of data for quality control and research. Health Inform J 2009; 15(4): 305-319.

14. Lenz R, Blaser R, Beyer M, Heger O, Biber C, Bäumlein M, Schnabel M. IT support for clinical pathways--lessons learned. Int J Med Inform. 2007 Dec;76 Suppl 3:S397-402. Epub 2007 Jun 12.

15. Courtney L, Gordon M, Romer L. A clinical path for adult diabetes. Diabetes Educ. 1997 Nov-Dec;23(6):664-71. 
16. U.S. National Cancer Institute 'Common Terminology Criteria for Adverse Events (CTCAE) and Common Toxicity Criteria (CTC)', http://ctep.cancer.gov/protocolDevelopment/electronic_applications/ctc.htm. (2010, accessed June 2011).

17. Matsumura Y, Kuwata S, Yamamoto Y, et al. Template-based data entry for general description in medical records and data transfer to data warehouse for analysis. Stud Health Technol Inform 2007; 129(Pt 1): 412-416.

18. Henry SB, Douglas K, Galzagorry G, Lahey A, Holzemer WL. A template-based approach to support utilization of clinical practice guidelines within an electronic health record. J Am Med Inform Assoc. 1998 May-Jun;5(3):237-44.

19. Los RK, van Ginneken AM, van der Lei J. OpenSDE: a strategy for expressive and flexible structured data entry. Int J Med Inform. 2005 Jul;74(6):481-90.

20. Rose EA, Deshikachar AM, Schwartz KL, Severson RK. Use of a template to improve documentation and coding. Fam Med. 2001 Jul-Aug;33(7):516-21.

21. Chen R, Enberg G, Klein GO. Julius--a template based supplementary electronic health record system. BMC Med Inform Decis Mak. 2007 May 2;7:10.

22. Prat N. A UML-based data warehouse design method. Decis Support Syst. 2006;42(3):1449. 
23. Grant A, Moshyk A, Diab H, et al. Integrating feedback from a clinical data warehouse into practice organisation. Int J Med Inform. 2006;75(3-4):232-9.

24.Rubin DL, Desser TS. A data warehouse for integrating radiologic and pathologic data. J Am Coll Radiol. 2008;5(3):210.

25. Wade TD, Hum RC, Murphy JR. A Dimensional Bus model for integrating clinical and research data. J Am Med Inform Assoc. 2011;18 Suppl 1:i96-102

26. Gordon BD, Asplin BR. Using online analytical processing to manage emergency department operations. Acad Emerg Med. 2004; 11(11):1206-12.

27. University Hospital Medical Information Network 'UMIN Clinical Trials Registry (UMIN-CTR)', http://www.umin.ac.jp/ctr/ (2005, accessed June 2011).

28. Bruix J, Sala M, Llovet JM. Chemoembolization for hepatocellular carcinoma. Gastroenterology 2004; 127(5 Suppl 1): S179-S188.

29. Yamanaka K, Hatano E, Narita M, et al. Comparative study of cisplatin and epirubicin in transcatheter arterial chemoembolization for hepatocellular carcinoma. Hepatol Res 2011; 41: 303-309.

30. Forner A, Ayuso C, Varela M, et al. Evaluation of tumor response after locoregional therapies in hepatocellular carcinoma: are response evaluation criteria in solid tumors reliable?. Cancer 2009; 115: 616-623. 
31. Kudo M, Kubo S, Takayasu K, et al. Response Evaluation Criteria in Cancer of the Liver (RECICL) proposed by the Liver Cancer Study Group of Japan (2009

Revised Version). Hepatol Res 2010; 40(7): 686-692.

32. Brown JM, Haining SA, Hale JM. Views on local data management in cancer clinical trials. Clin Oncol (R Coll Radiol) 1997; 9(6): 403-406.

33. Tunis SR, Stryer DB, Clancy CM. Practical clinical trials: increasing the value of clinical research for decision making in clinical and health policy. JAMA 2003; 290(12): 1624-1632.

34. Joo JH, Morales KH, de Vries HF, Gallo JJ. Disparity in use of psychotherapy offered in primary care between older african-american and white adults: results from a practice-based depression intervention trial. J Am Geriatr Soc. 2010 Jan;58(1):154-60.

35. Tunis SR, Benner J, McClellan M. Comparative effectiveness research: Policycontext, methods development and research infrastructure. Stat Med. 2010 Aug 30; 29(19):1963-76.

36. Rostami R, Nahm M, Pieper CF: What can we learn from a decade of database audits? The Duke Clinical Research Institute experience, 1997--2006. Clin Trials. 2009;6(2):141-50, 
37. Pan JJ, Nahm M, Wakim P, et al. A centralized informatics infrastructure for the

National Institute on Drug Abuse Clinical Trials Network. Clin Trials 2009; 6(1): $67-75$.

38. WHO 'International Classification of Diseases (ICD)', http://www.who.int/classifications/icd/en/ (2007, accessed June 2011).

39. MedDRA MSSO 'MedDRA MSSO’, http://www.meddramsso.com/ (2011, accessed June 2011).

40. U.S. National Library of Medicine 'SNOMED Clinical Terms', http://www.nlm.nih.gov/research/umls/Snomed/snomed_main.html (2009, accessed June 2011).

41. Clinical Data Interchange Standards Consortium (CDISC) 'Operational Data Model', http://www.cdisc.org/odm (2011, accessed June 2011).

42. Clinical Data Interchange Standards Consortium (CDISC) 'Study Data Tabulation Model', http://www.cdisc.org/sdtm (2011, accessed June 2011).

43.US. Food and Drug Administration 'CFR - Code of Federal Regulations Title 21', http://www.accessdata.fda.gov/scripts/cdrh/cfdocs/cfcfr/cfrsearch.cfm?cfrpart=11 (2011, accessed Jan 2012). 
Table 1. The Screen to Record Data for EMRs and the Data Check Screen.

\begin{tabular}{|c|c|c|}
\hline \multicolumn{2}{|c|}{ Screen to Record Data for EMRs } & \multirow{2}{*}{ Edit Check Screen } \\
\hline Template Name & Item Name & \\
\hline \multirow{6}{*}{ Eligibility Criteria } & Informed consent & \multirow{6}{*}{$\begin{array}{l}\text { List of Cases } \\
\text { Case Registration Form }\end{array}$} \\
\hline & Registration Number & \\
\hline & Date of Consent & \\
\hline & Result of Allocation & \\
\hline & Selection Criteria $1-8$ & \\
\hline & Exclusion Criteria 1-10 & \\
\hline \multirow{7}{*}{ Target Lesion } & Number & \multirow{24}{*}{ Case Report Form } \\
\hline & Tumour Location & \\
\hline & Size $(\max )$ & \\
\hline & Incipient TACE/non-Incipient & \\
\hline & TACE & \\
\hline & Vascular Invasion & \\
\hline & Frequency of TACE & \\
\hline \multirow{9}{*}{ Protocol Treatment } & Date of Protocol Treatment & \\
\hline & Size $(\max )$ & \\
\hline & Total Dose & \\
\hline & Number & \\
\hline & Total Dose for TACE & \\
\hline & Tumour Location & \\
\hline & Addition of RFA/PEIT & \\
\hline & Anticancer Drug & \\
\hline & Infusional Therapy & \\
\hline \multirow{4}{*}{$\begin{array}{l}\text { Efficacy of } \\
\text { Treatment (EASL) } \\
\text { Efficacy of } \\
\text { Treatment } \\
\text { (RECICL) }\end{array}$} & Efficacy of Treatment & \\
\hline & Recurrence Form & \\
\hline & Plan of Treatment & \\
\hline & Other & \\
\hline \multirow{4}{*}{ Adverse Event } & Abdominal Pain & \\
\hline & Cause of Abdominal Pain & \\
\hline & Diarrhoea & \\
\hline & Cause of Diarrhoea & \\
\hline
\end{tabular}




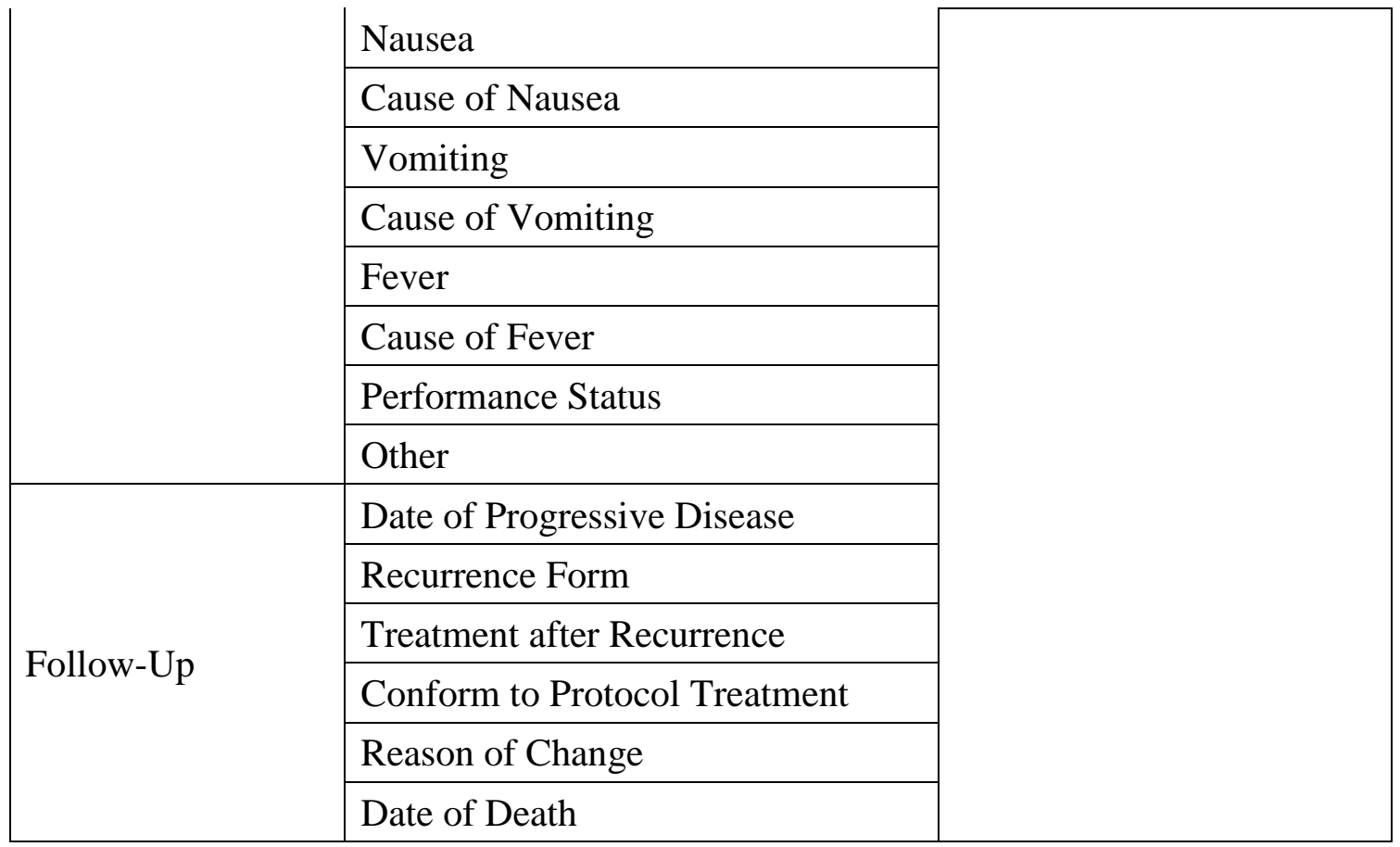

EASL: European Association for the Study of the Liver; EMR: electronic medical

record; PEIT: percutaneous ethanol injection therapy; RECICL: Response Evaluation

Criteria in Cancer of the Liver; RFA: radiofrequency ablation; TACE: transcatheter arterial chemoembolisation. 


\section{Figure captions}

\section{Figure 1.}

Our new model of the clinical trial process.

\section{Figure 2.}

Electronic medical record (EMR) and the EMR template. The template is opened from within our EMR system. The template converts narrative information from progress notes, thereby making the information available as analytical data.

\section{Figure 3.}

The List of Cases and the Case Report Form. The Case Report Form can be consulted from the List of Case Registration Forms. The Case Report Form summarises all the templates on one screen so that all information can be assessed at a glance. EASL: European Association for the Study of the Liver; EMR: electronic medical record; RECICL: Response Evaluation Criteria in Cancer of the Liver.

\section{Figure 4.}

The pilot study revealed many advantages over a conventional clinical trial process, i.e., unnecessary to transfer information from medical records to the case report form, no need for source data verification at the participating site, unnecessary to transmit the case report form from the participating site to the coordinating centre, and unnecessary 
to re-enter data into the clinical data management system (CDMS) from the paper case report form. In addition, data management in the coordinating centre was replaced with edit checks initiated by a local data manager at the participating site. CRF: case report form; CDMS: clinical data management system; DWH: data warehouse. 
Figure 1.






\section{Figure 2.}

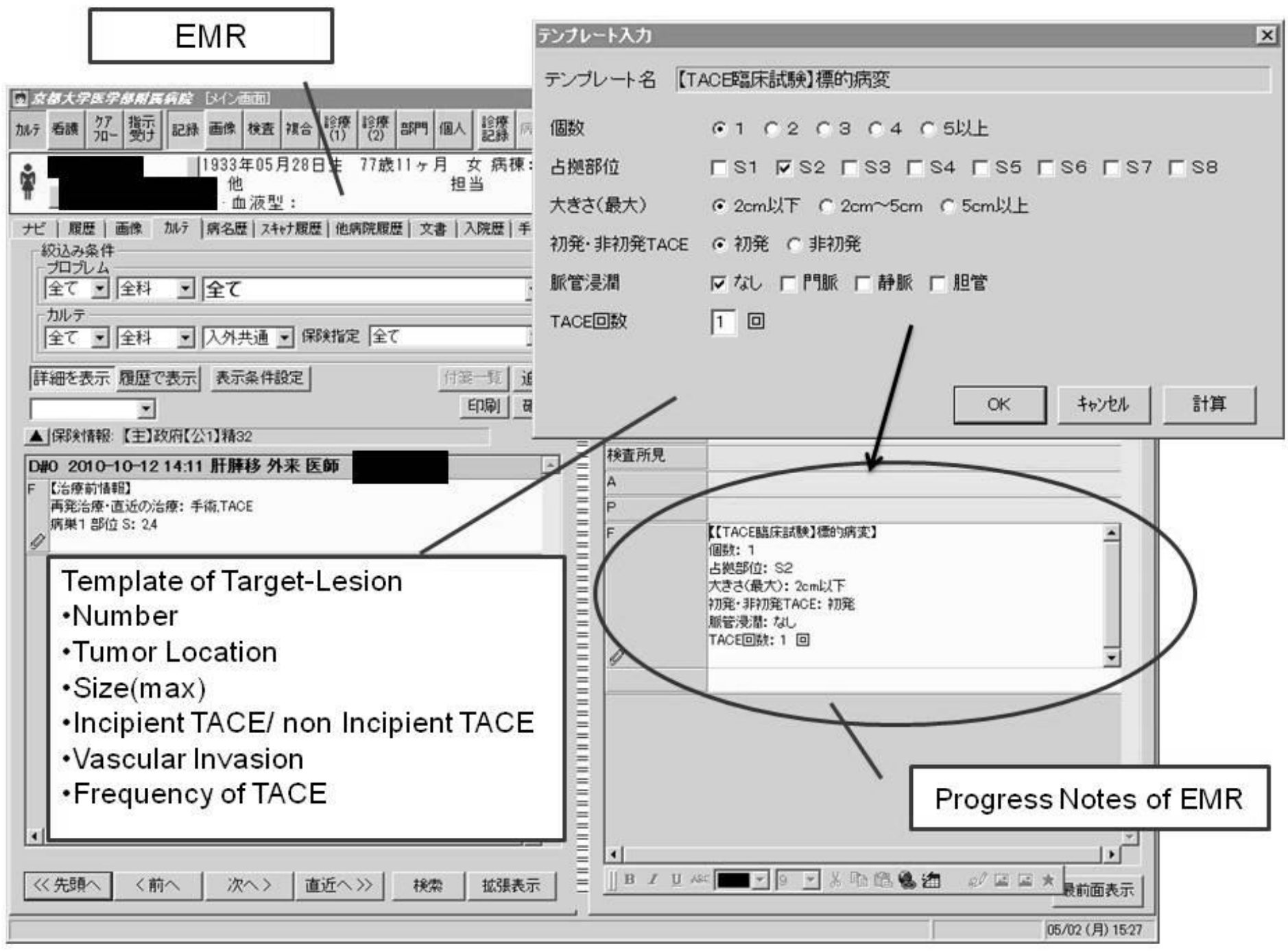




\section{Figure 3.}

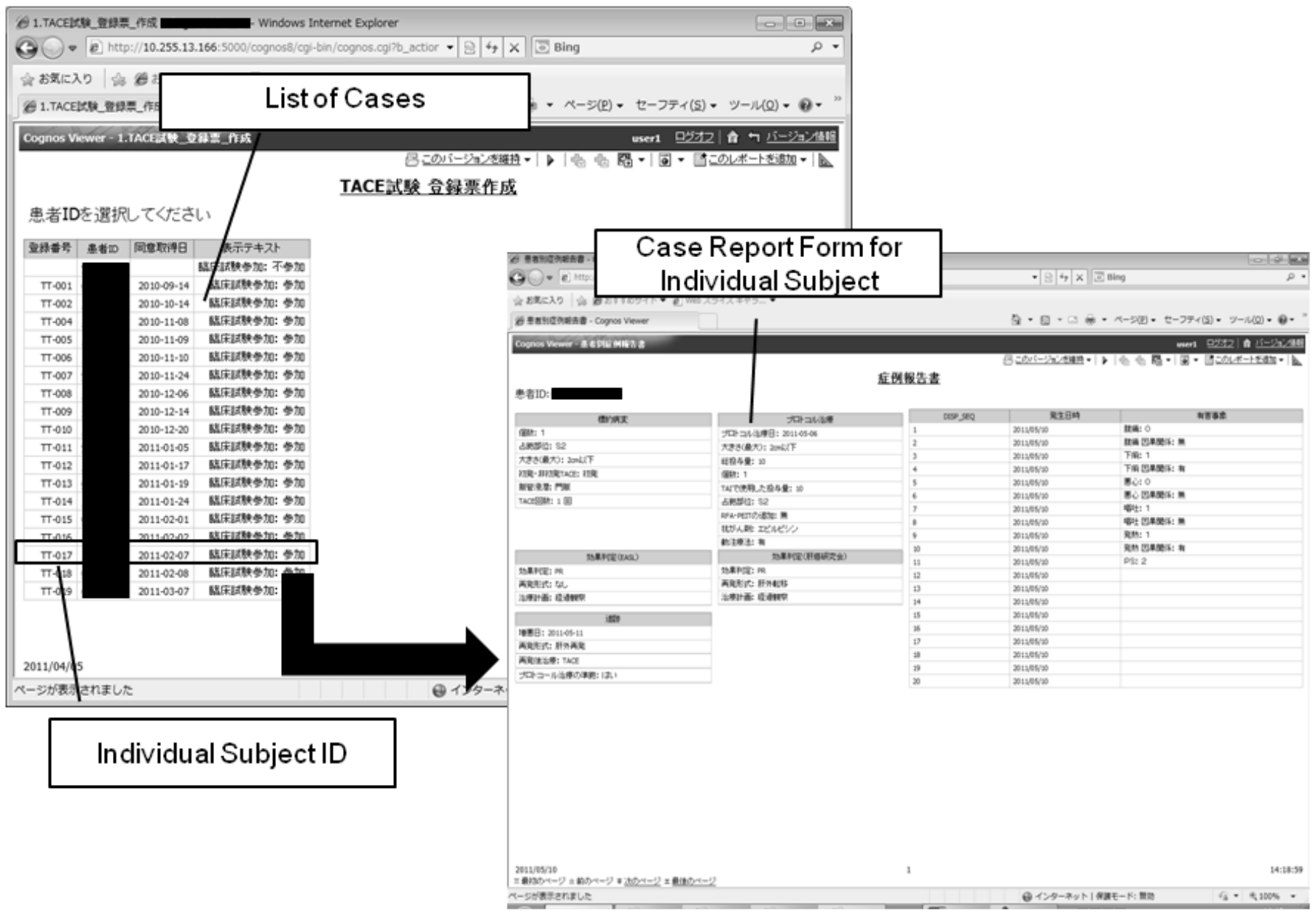


Figure 4.

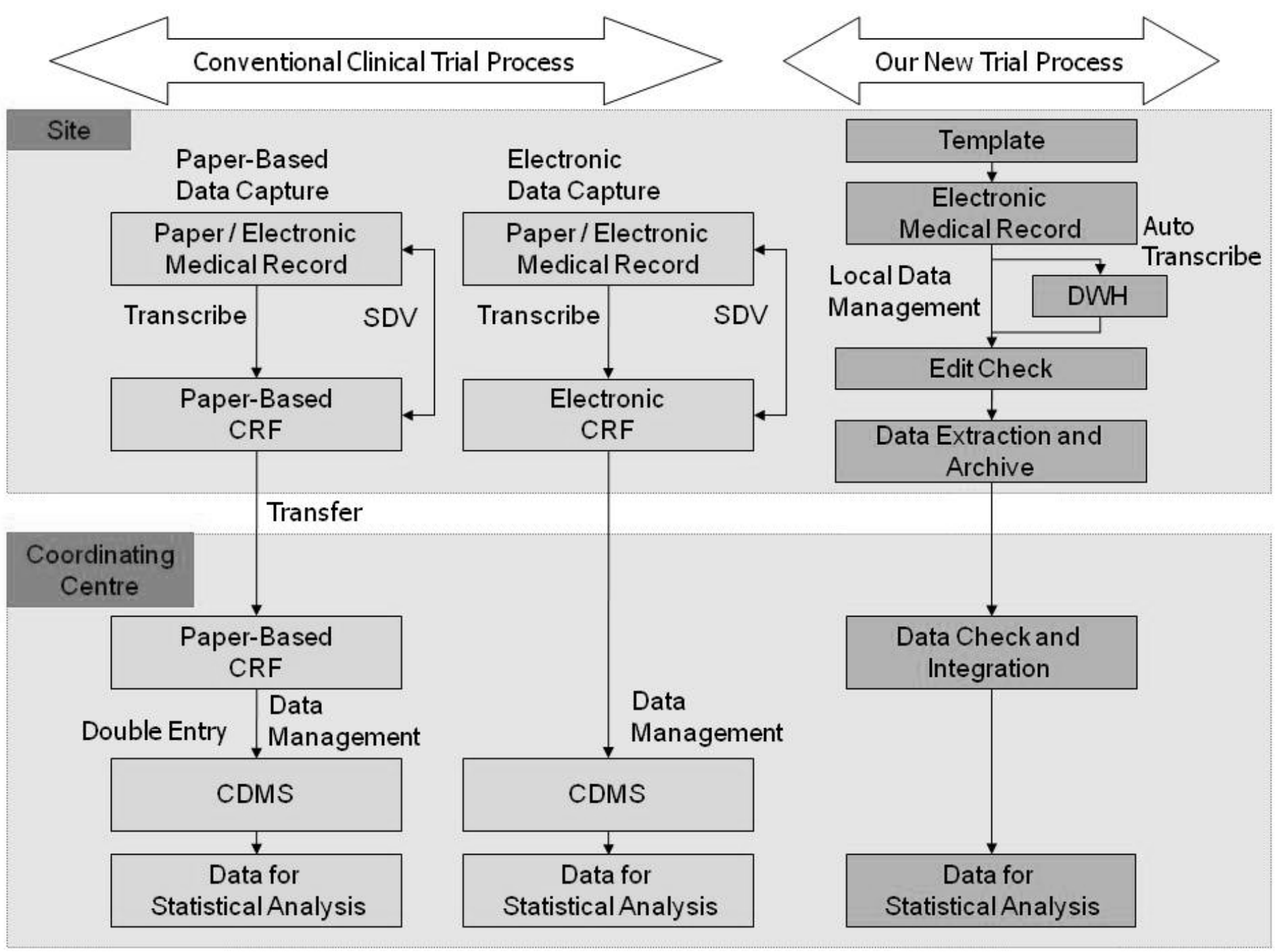




\section{List of abbreviations}

CDISC: clinical data interchange standards consortium;

CDMS: clinical data management system;

CRF: case report form;

CSV: comma separated values;

CTCAE: common terminology criteria for adverse events;

DWH: data warehouse;

EASL: european association for the study of the liver;

EDC: electronic data capture;

EMR: electronic medical records;

ERS: electronic medical records retrieval system;

eSDI: electronic source data interchange;

eSource: electronic source data;

HTML: hypertext markup language;

ICD: international classification of diseases;

MedDRA: medical dictionary for regulatory activities;

ODM: operational data model;

OLAP: online analytical processing; 
PDF: portable document format;

PEIT: percutaneous ethanol injection therapy;

RECICL: response evaluation criteria in cancer of the liver;

RFA: radiofrequency ablation;

SDTM: study data tabulation model;

SDV: source data verification;

SNOMED: systematized nomenclature of medicine;

TACE: transcatheter arterial chemoembolization;

XML: extensible markup language; 